\title{
Ordering mechanisms in triblock copolymers
}

\author{
P. Maniadis, ${ }^{1,2,3}$ R. B. Thompson, ${ }^{1}$ K. Ø. Rasmussen, ${ }^{1}$ and T. Lookman ${ }^{1}$ \\ ${ }^{1}$ Theoretical Division, Los Alamos National Laboratory, Los Alamos, New Mexico 87545, USA \\ ${ }^{2}$ LADIR-CNRS, UMR 7075, Universitè Pierre et Marie Curie, 2 rue Henry Dunant, 94320 Thiais, France \\ ${ }^{3}$ Laboratoire Léon Brillouin (CEA-CNRS), CEA Saclay, 91191 Gif-sur-Yvette Cedex, France
}

(Received 24 November 2003; published 17 March 2004)

\begin{abstract}
The ordering mechanisms for an $A B C$ triblock copolymer system are studied using self-consistent field theory. We find a two-phase mechanism, similar to what has been suggested experimentally (two-step mechanism). Analysis of free energy components shows that the two-phase process comes about through a competition between stretching energy and interfacial energy. The mechanism is found to be sufficiently robust so as to make it potentially useful for device applications.
\end{abstract}

DOI: 10.1103/PhysRevE.69.031801

PACS number(s): 83.80.Uv, 82.35.Jk, 61.25.Hq

\section{INTRODUCTION}

Block copolymers are macromolecules composed of dissimilar homopolymers covalently tethered together. In particular, $A B$ diblock copolymer melts have received much attention, and over the past few years our understanding of the microphase-separated morphologies has significantly increased [1-3]. In the microphase-separated state, the connectivity of $A$ and $B$ blocks requires the molecules to stretch in order to form $A$ - and $B$-rich domains. The result is that the domain sizes cannot exceed molecular length scales, and many $A / B$ domain interfaces form. In an attempt to compensate for the stretching, spontaneous curvature of the interface occurs. This tendency is enhanced for increasingly asymmetric molecules resulting in the well-known array of morphologies ranging from lamellar to cylindrical to spheres. At the boundary between the lamellar and cylindrical phases a number of complex morphologies with favorable interfacial curvature can exist, however, the gyroid structure is the only stable phase because of a lower packing frustration [4] than other phases.

Parallel with these advances in the understanding of the morphological behavior of diblock copolymers, several preliminary studies have illustrated that the behavior becomes far more complex and elaborate with the addition of a third block [5]. The $A B C$ triblock copolymer is architecturally only slightly more complicated than the $A B$ diblock copolymer, but generates a phase diagram so complex that to date it has been only marginally explored. $A B C$ triblock copolymer morphologies must minimize the stretching energy from three different domains and the interfacial energy from up to three distinct interfaces. Additionally, there are geometrical constraints on the domain sequence requiring that a $B$-rich domain be in contact with both $A$ - and $C$-rich domains. This complexity results, in contrast to the four distinct morphologies offered by $A B$ diblock copolymers, in a far richer palette of morphologies [5]. Increasing our understanding of ordering and control of $A B C$ triblock morphological behavior provides a powerful alternative to the use of simple diblock systems for nanopatterning [6] and nanoparticle scaffolding applications [7], for example.

Preliminary studies of complex ordering in triblock systems were experimentally undertaken by Yamauchi et al. [8,9], who examined polyisoprene-block-poly ( $\mathrm{D}_{8}$-styrene)-block-poly(vinyl methyl ether) triblock terpolymers and found that ordering may proceed via a two-phase mechanism. Segregation of one species from the other two occurred first, followed by complete microphase separation between all three chemical species [10].

With this in mind, we investigate theoretically the ordering process of a triblock copolymer in two cases-one case where the three components of a triblock simultaneously segregate from each other and the other case where two of the components $(A$ and $C$ ) mix while segregating away from the third $(B)$. Using self-consistent field theory (SCFT) [2], we determine the regimes where these two cases occur and find that it very much depends on the morphologies considered. Also, we clarify the physical concepts that control the selection between the two cases.

\section{THEORY}

Since SCFT has already been applied to $A B C$ triblock copolymers by a number of authors [11-16] we limit ourselves to briefly summarizing the theory. More details on block copolymer SCFT can be found in the recent review by Matsen [2].

Consider a volume $V$ containing an incompressible melt of $n A B C$ triblock copolymer molecules, each of polymerization $N$, based on a common segment volume $\rho_{0}^{-1}$. The statistical segment lengths of the $A, B$, and $C$ segments are given by $a_{A}, a_{B}$, and $a_{C}$, respectively. The SCFT free energy for this system is then [15]

$$
\begin{aligned}
\frac{F}{n k_{B} T}= & -\ln \left(\frac{Q}{V}\right)+\frac{1}{V} \int d \mathbf{r}\left\{\chi_{A B} N \varphi_{A}(\mathbf{r}) \varphi_{B}(\mathbf{r})\right. \\
& +\chi_{B C} N \varphi_{B}(\mathbf{r}) \varphi_{C}(\mathbf{r})+\chi_{A C} N \varphi_{A}(\mathbf{r}) \varphi_{C}(\mathbf{r}) \\
& -w_{A}(\mathbf{r}) \varphi_{A}(\mathbf{r})-w_{B}(\mathbf{r}) \varphi_{B}(\mathbf{r})-w_{C}(\mathbf{r}) \varphi_{C}(\mathbf{r}) \\
& \left.-\xi(\mathbf{r})\left[1-\varphi_{A}(\mathbf{r})-\varphi_{B}(\mathbf{r})-\varphi_{B}(\mathbf{r})\right]\right\} .
\end{aligned}
$$

In Eq. (1), $\varphi_{A}(\mathbf{r}), \varphi_{B}(\mathbf{r})$, and $\varphi_{C}(\mathbf{r})$ are the local segment (monomer) densities, and $w_{A}(\mathbf{r}), w_{B}(\mathbf{r})$, and $w_{C}(\mathbf{r})$ are the conjugate chemical potential fields. $\xi(\mathbf{r})$ is a field that enforces incompressibility. $Q$ is the partition function of a 
single triblock molecule subject to the $A, B$, and $C$ chemical potential fields. $T$ is the temperature, and $k_{B}$ is Boltzmann's constant. The degrees of incompatibility between various monomer types are represented by the Flory-Huggins parameters $\chi_{A B}, \chi_{B C}$, and $\chi_{A C}$.

SCFT is implemented by varying the free energy functional (1) with respect to each of the seven functions $\varphi_{A}(\mathbf{r})$, $\varphi_{B}(\mathbf{r}), \varphi_{C}(\mathbf{r}), w_{A}(\mathbf{r}), w_{B}(\mathbf{r}), w_{C}(\mathbf{r})$, and $\xi(\mathbf{r})$. The resulting set of equations can be found in Ref. [15]. These equations are solved numerically and self-consistently to find the equilibrium morphology for a given set of system parameters.

Three of the parameters are the Flory-Huggins segregation variables. Since in Eq. (1), these segregation parameters always appear as a product with the degree of polymerization, we will take $\chi_{A B} N, \chi_{B C} N$, and $\chi_{A C} N$ as the independent quantities from now on. In addition to the three segregation parameters, the volume fractions of the monomer species must be known. Let these be denoted by $f_{A}, f_{B}$, and $f_{C}$. Since incompressibility must be satisfied, we have that

$$
f_{A}+f_{B}+f_{C}=1
$$

and so only two of these three are independent. The ratios of the statistical segment lengths provide two more free parameters, so overall the triblock system will be fully specified by seven independent parameters.

\section{RESULTS AND DISCUSSION}

The application of SCFT to a triblock copolymer system allows us to determine the possible morphologies upon varying the seven independent parameters. We will assume throughout this work that the statistical segment lengths for the various species all are the same, so that $a \equiv a_{A}=a_{B}$ $=a_{C}$. This leaves us with only five parameters. Without loss of generality for the phenomenon we are investigating we will also limit our attention to cases where $\chi_{A B} N=\chi_{B C} N$ $=50$. Further, the $A$ and $C$ block sizes will be taken as equal so that $f_{A}=f_{C} \equiv f_{A / C}$, leaving only two parameters. Let us first consider lamellar morphologies. Choosing $f_{A / C}=0.25$ and $\chi_{A C} N$ large, the equilibrium morphology is lamellar with distinct $A, B$, and $C$ domains, as shown by the solid curves in Fig. 1.

The transition between the situations depicted in Figs. 1 and 2 upon lowering $\chi_{A C} N$ is smooth, and constitutes a miniature order-disorder transition (ODT) (see Fig. 3) between the $A$ and $C$ blocks of the polymers. This prediction is similar to the experimental results of Yamauchi et al. [8,9] where a two-step (two-phase) microphase separation was observed in polyisoprene-block-poly $\left(\mathrm{D}_{8}\right.$-styrene)-block-poly(vinyl methyl ether) triblock terpolymers.

This sub-ODT can be understood by examining the free energy components. SCFT formulas for the internal energy, translational entropy, and conformational entropies can be found for diblock systems in Ref. [3]. For triblocks, they can be written as

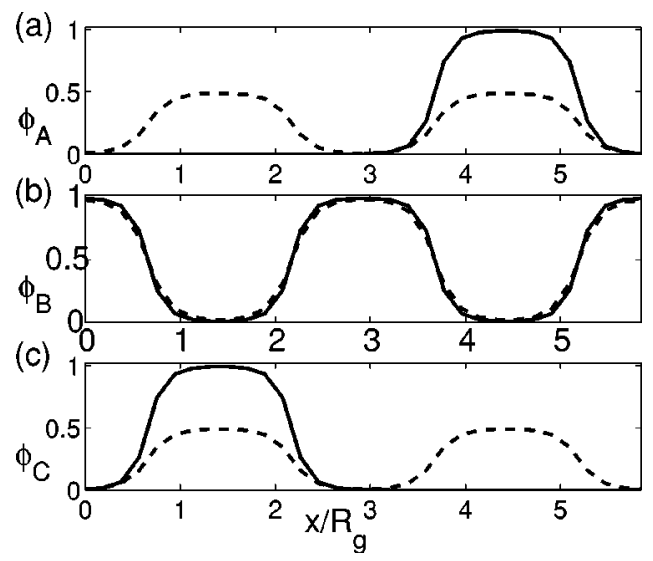

FIG. 1. Density profiles for an $A B C$ triblock copolymer melt in a lamellar phase with $\chi_{A C} N=50$. (a) $A$ segment distribution. (b) $B$ segment distribution. (c) $C$ segment distribution. The solid curves are the equilibrium distributions, while the dashed curves are distributions where equal amounts of $A$ and $C$ segments are artificially enforced everywhere [17].

$$
\begin{gathered}
\frac{U}{n k_{B} T}=\frac{\chi_{A B} N}{V} \int d \mathbf{r} \varphi_{A}(\mathbf{r}) \varphi_{B}(\mathbf{r})+\frac{\chi_{B C} N}{V} \int d \mathbf{r} \varphi_{B}(\mathbf{r}) \varphi_{C}(\mathbf{r}) \\
+\frac{\chi_{A C} N}{V} \int d \mathbf{r} \varphi_{A}(\mathbf{r}) \varphi_{C}(\mathbf{r}), \\
\quad \frac{-S_{E}}{n k_{B}}=\frac{1}{V} \int d \mathbf{r} \rho(\mathbf{r}) \ln \rho(\mathbf{r}) \\
\frac{-S_{A}}{n k_{B}}=-\frac{1}{V} \int d \mathbf{r}\left[\rho(\mathbf{r}) \ln q\left(\mathbf{r}, f_{A}\right)+w_{A}(\mathbf{r}) \varphi_{A}(\mathbf{r})\right] \\
\frac{-S_{C}}{n k_{B}}=-\frac{1}{V} \int d \mathbf{r}\left[\rho(\mathbf{r}) \ln q^{\dagger}\left(\mathbf{r}, f_{A}+f_{B}\right)+w_{C}(\mathbf{r}) \varphi_{C}(\mathbf{r})\right]
\end{gathered}
$$

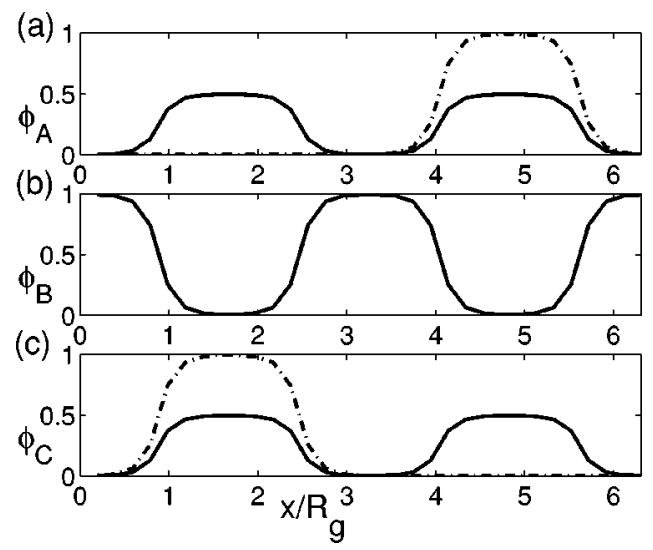

FIG. 2. Density profiles for an $A B C$ triblock copolymer melt in a lamellar phase with $\chi_{A C} N=0.1$. The solid curves are the equilibrium distributions, while the dot-dashed curves are distributions where $A$ and $C$ segments are artificially forced to be segregated [18]. 

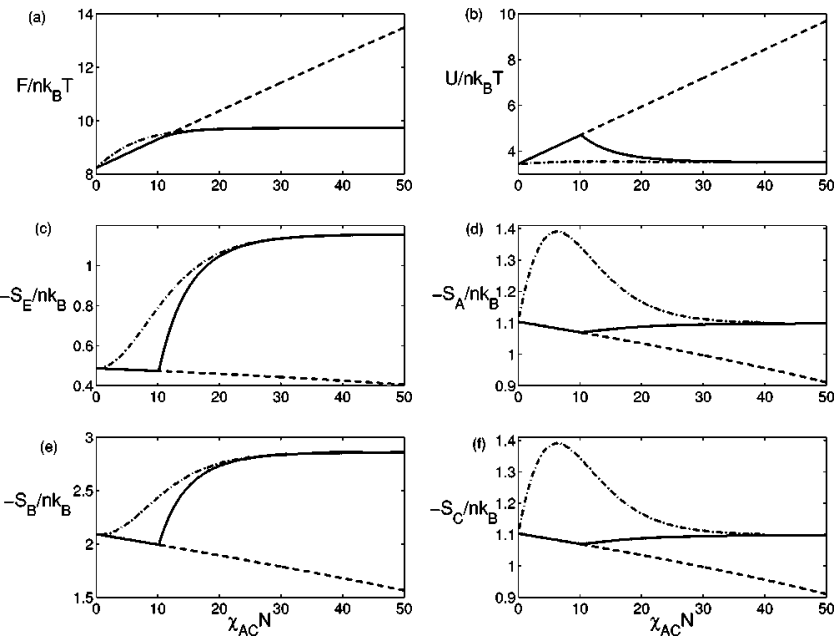

FIG. 3. Free energy of an $A B C$ triblock copolymer with $f_{A / C}$ $=0.25$ as a function of $\chi_{A C} N$. (a) The total free energy. (b)-(f) Components of the free energy: internal energy contribution, translational entropy contribution, $A, B$, and $C$ block conformational entropy contributions to the free energy, respectively. The solid curves are the equilibrium energies. Dashed curves correspond to an artificial enforcement of equal $A$ and $C$ segment densities everywhere [17]. The dashed-dotted curves correspond to distributions where $A$ and $C$ are artificially forced to be segregated [18].

$$
\frac{-S_{B}}{n k_{B}}=\frac{F}{n k_{B} T}-\frac{U}{n k_{B} T}-\left[\frac{-S_{E}}{n k_{B}}+\frac{-S_{A}}{n k_{B}}+\frac{-S_{C}}{n k_{B}}\right],
$$

where $\rho(\mathbf{r})=(V / Q) q(\mathbf{r}, 1)$ is the distribution of a single point on the triblock, in this case chosen to be the $C$ end point.

Thus Eq. (4) gives the translational entropy contribution to the free energy. Likewise, Eqs. (5) and (6) give the conformational entropy contributions of the $A$ and $C$ end blocks of the polymer. The internal energy contribution is given by Eq. (3), and is a measure of the number of contacts between dissimilar monomers. The conformational entropy contribution of the middle $B$ block can be found by subtracting all the other contributions from the total free energy, as shown in Eq. (7). Figure 3(a) shows the free energy as a function of $A C$ segregation. The solid line is the equilibrium free energy, while the dashed curve corresponds to a situation in which the $A$ and $C$ monomers are artificially forced to mix in equal amounts everywhere [17], as shown by the dashed profile in Fig. 1. Similarly, the dot-dashed curve denotes a morphology where the $A$ and $C$ monomers are artificial forced to be segregated [18]. This morphology is depicted in Fig. 2 by the (a)

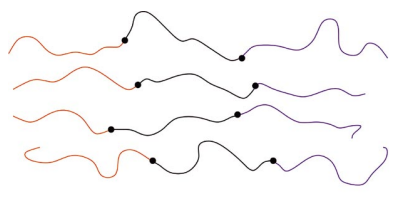

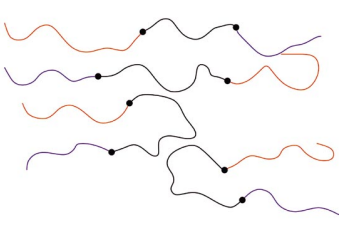

(b)
FIG. 4. (Color) Possible configurations of an $A B C$ triblock copolymer. (a) $A$ and $C$ are segregated. (b) $A$ and $C$ are mixed.

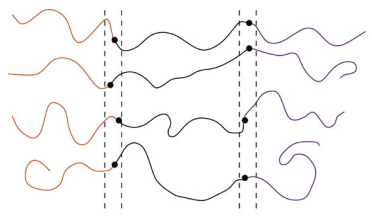

(a)

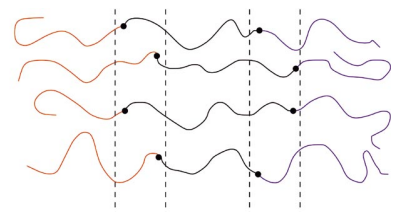

(b)
FIG. 5. (Color) Effect of the interfacial width on an $A B C$ triblock copolymer. The black dots denote junction points and the dashed lines give the approximate positions of the edges of the interfaces. (a) A narrow interface. (b) A wide interface.

dot-dashed profile. One can see from Fig. 3(a) that the completely mixed state is preferred for $A C$ segregations of $\chi_{A C} N \lesssim 10.2$. This is only slightly less than the segregation value $(\chi N \simeq 10.4)$ where the ODT occurs for symmetric diblocks. At $\chi_{A C} N=0$, all the curves terminate at the same point, since $A$ and $C$ monomers are indistinguishable so that there is no difference between mixed and segregated states. In contrast to the three distinct regions seen in Fig. 1 for high $\chi_{A C} N$ (the solid curves), if $\chi_{A C} N$ is very small or zero, a lamellar structure with only two distinct regions is formed, as shown by the solid curves in Fig. 2. Panels (b)-(f) of Fig. 3 show the components of the free energy. From these we can see that for low segregations, the internal energy contribution $U$ of the mixed state suffers as a result of the large numbers of $A C$ contacts. This is more than compensated for, however, by the significantly lower entropic contributions. The conformational entropy contributions $S_{A}, S_{B}$, and $S_{C}$ shown in panels (d), (e), and (f), respectively, benefit because there are more configurations available to the molecules in the mixed state. As illustrated in Fig. 4(a), the segregated state forces the molecules to bridge between the $A$ and $C$ domains.

In the mixed state shown in panel (b), the molecules may be oriented in either the $A B C$ or $C B A$ directions, and may either loop or bridge. The translational entropy also benefits from being in a mixed phase since the interfacial width is larger in the mixed state than in the segregated, as seen in Fig. 1(b) [19]. The advantage of a wide interface is illustrated in Fig. 5, where a narrow interface configuration [panel (a)] is contrasted to a wide interface [panel (b)]. The top two molecules in (b) show that there are more positions (a)

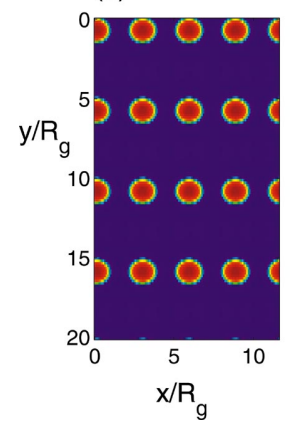

(b)

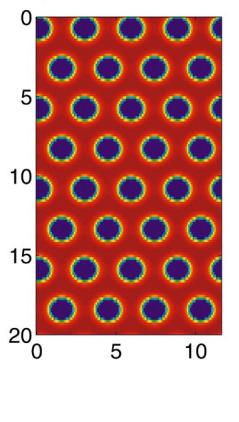

(c)

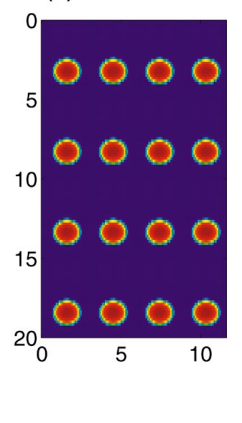

FIG. 6. (Color) Density distributions for an $A B C$ triblock melt in a cylindrical phase with $\chi_{A C} N=50$. (a) $A$ segment distribution. (b) $B$ segment distribution. (c) $C$ segment distribution. 
(a)

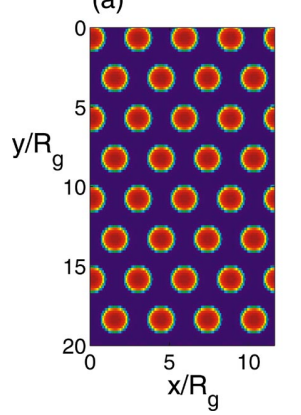

(b)

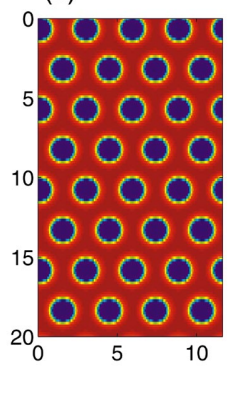

(c)

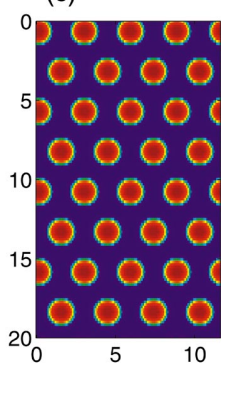

FIG. 7. (Color) Density distributions for an $A B C$ triblock melt in a hexagonally packed cylindrical phase with $\chi_{A C} N=0.6$. (a) $A$ segment distribution. (b) $B$ segment distribution. (c) $C$ segment distribution.

available in a wide interface system than in a narrow interface system. The conformational entropy of the $B$ block benefits in the same manner.

The bottom two molecules of Fig. 5(b) show that the $B$ block can stretch or contract more in a wide interface than in a narrow one. The impact of the interfacial width accounts for the qualitative similarity between the $S_{E}$ curves and the $S_{B}$ curves. For high $A C$ segregations, the internal energy wins out over the entropies. The dashed-dotted curve in Fig. 3 demonstrates that the segregated state is preferred from the point of view of the internal energy $U$. The advantages of greater entropic freedom are now overpowered. Thus we see that the sub-ODT results from the standard competition between interfacial tension (internal energy) and stretching energy (entropy) known as packing frustration [4], even though a global lamellar phase is maintained throughout.

The sub-ODT is not only a feature of the lamellar phase. Our calculations show that it is a general feature of the tri- (a)
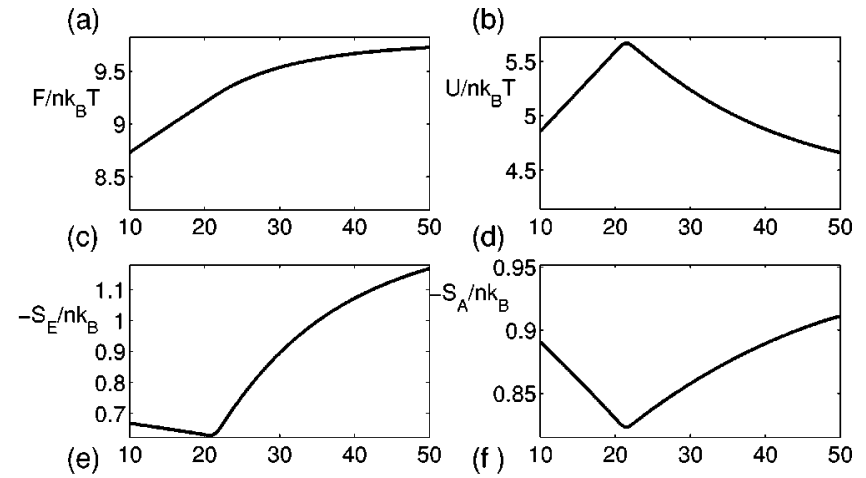

(d)

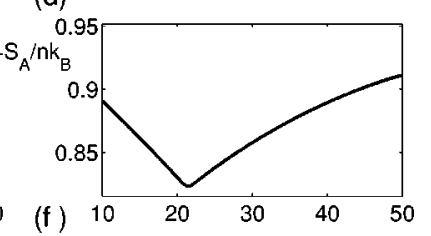

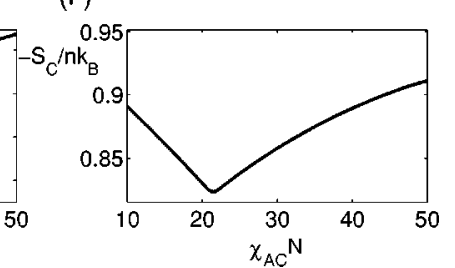

FIG. 8. Free energy of an $A B C$ triblock copolymer with $f_{A / C}$ $=0.14$ as a function of $\chi_{A C} N$. (a) The total free energy. (b)-(f) Components of the free energy: internal energy contribution, translational entropy contribution, $A, B$, and $C$ block conformational entropy contributions to the free energy, respectively.
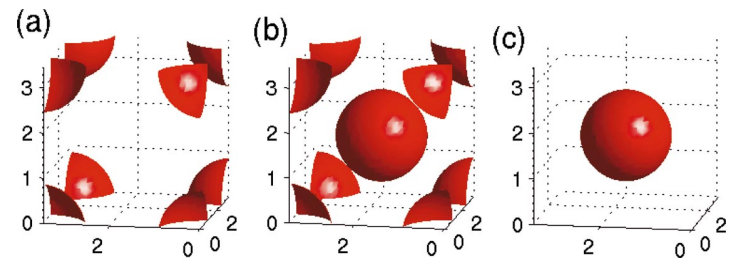

FIG. 9. (Color) Density distributions for an $A B C$ triblock melt in a body-centered-cubic (bcc) spherical phase with $\chi_{A C} N=50$ (only a single unit cell is shown). (a) $A$ segment distribution. (b) $B$ segment distribution. (c) $C$ segment distribution. The isosurfaces are at $\varphi_{A}=0.5, \varphi_{B}=0.5$, and $\varphi_{C}=0.5$, respectively.

block phase diagram. Figure 6 shows a system for which $f_{A / C}=0.14$ and $\chi_{A C} N=50$. A cylindrical morphology is seen where the $A$ and $C$ domains are distinct. This may be contrasted with the case where $\chi_{A C} N=0.6$, shown in Fig. 7 , where the $A$ and $C$ monomers mix.

The free energy components for this cylindrical system are shown in Fig. 8. From the components of the free energy we can see that the transition in this cylindrical morphology occurs at $\chi_{A C} N \simeq 21.4$, which is at a much higher segregation than in the lamellar case. It is therefore clear that this morphology forces the two, lengthwise symmetric, components ( $A$ and $C$ ) to mix (to be in the disordered regime) up to very high degree of segregation.

Similarly, Figs. 9 and 10 show the high $\left(\chi_{A C} N=50\right)$ and low $\left(\chi_{A C} N=10.5\right)$ segregation morphologies, respectively, for $f_{A / C}=0.12$.

Figure 11 shows the free energy components for the spherical morphologies, and we see that the transition occurs at $\chi_{A C} \simeq 24.0$ in this case.

\section{CONCLUSIONS}

$A B C$ triblock copolymers were examined using SCFT with a focus on the effects of varying the end block segregation, $\chi_{A C} N$. For high $\chi_{A C} N$, three distinct lamellar regions, corresponding to $A, B$, and $C$ monomers, were found. For low $\chi_{A C} N$, only two distinct domains were found, corresponding to $B$ monomers, and a mix of $A$ and $C$ monomers. This is an $A C$ ODT embedded within a $B$ lamellar morphology. Thus we find it is possible to have a two-phase separation process in $A B C$ triblock copolymers: $A B C$ disordered $\rightarrow A C$ disordered $/ B$ ordered $\rightarrow A / B / C$ ordered. This result is similar to the experimental observations of Yamauchi et al.

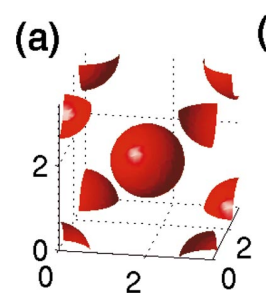

(b)

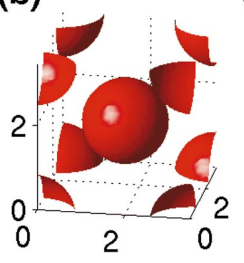

(c)

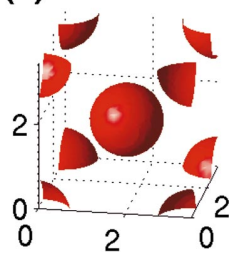

FIG. 10. (Color) Density distributions for an $A B C$ triblock melt in a bcc spherical phase with $\chi_{A C} N=10.5$. (a) $A$ segment distribution. (b) $B$ segment distribution. (c) $C$ segment distribution. The isosurfaces are at $\varphi_{A}=0.4, \varphi_{B}=0.4$, and $\varphi_{C}=0.4$, respectively. 
(a)

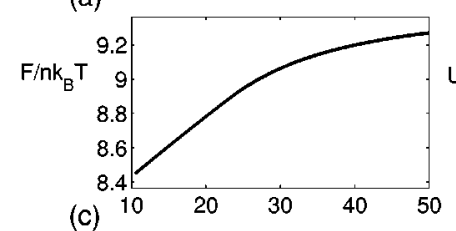

(b)
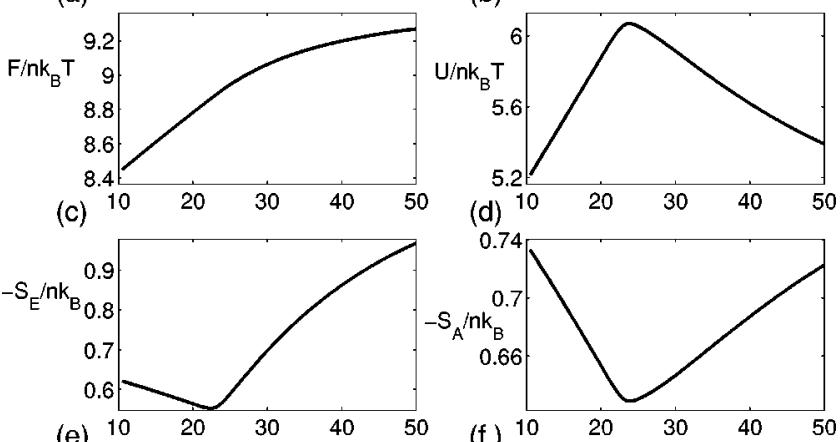

(e)
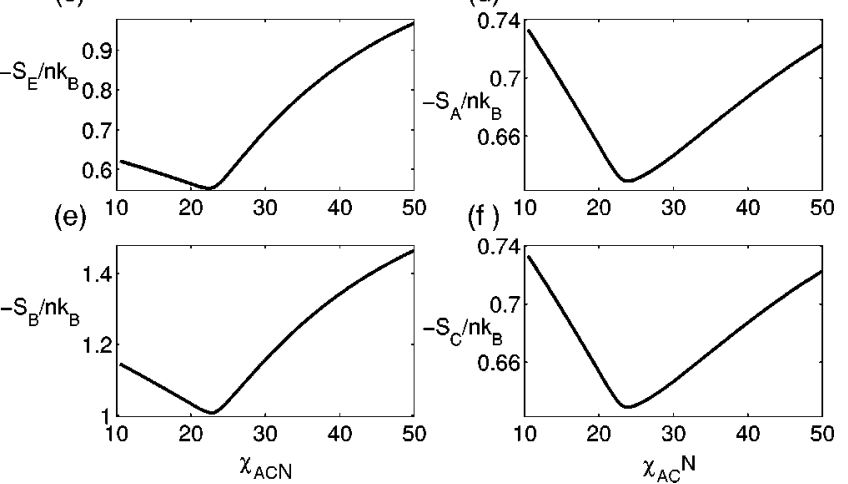

(f) 10

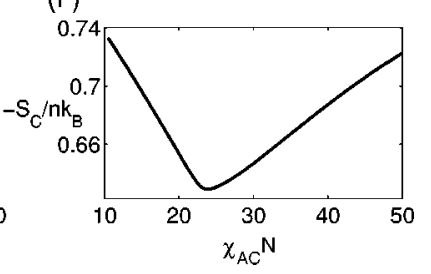

FIG. 11. Free energy of an $A B C$ triblock copolymer with $f_{A / C}$ $=0.12$ as a function of $\chi_{A C} N$. (a) The total free energy. (b)-(f) Components of the free energy: internal energy contribution, translational entropy contribution, $A, B$, and $C$ block conformational entropy contributions to the free energy, respectively.

$[8,9]$. Analysis of the free energy components shows that this sub-ODT arises from the standard competition between interfacial tension and stretching energy.

It is also interesting to note the similarity between the phase transition we observe and liquid-liquid phase transitions that recently have been observed in substances such as carbon [20] and phosphorus [21]. In these phase transitions the structure of the liquid changes through a first-order phase transition and thus an entropy change is observed, similar to the cases discussed here. However, an essential aspect of these liquid-liquid phase transition is the change in density, which is absent in the transitions we discuss.

Our calculations show the sub-ODT is present not just in the lamellar phase, but also in the cylindrical and spherical phases. We also found that the increased interfacial curvature of the latter phases forces the end blocks to mix to a degree of dissimilarity above what is seen for diblocks. That is, the curvature of the morphology effectively makes the end blocks much more miscible. Further, it is likely that the twophase ordering process is a general feature of the $A B C$ triblock copolymer phase diagram, occurring for all morphologies. For block copolymers with more distinct chemical species, such as $A B C D$ tetrablocks, or $A B C D E$ pentablocks, one could expect multiphase ordering as opposed to the triblock two-phase ordering.

In either two-phase or multiphase ordering, the selfassembly of one block could be controlled without affecting the self-assembly of the other blocks. This could be important in device applications if one block was assigned some functionality. Computational runs that we have not reported here show that the two-phase ordering process still occurs when assuming some temperature mechanism for varying $\chi_{A C} N$ that also affects $\chi_{A B} N$ and $\chi_{B C} N$. The phenomenon seems robust enough therefore for device application.

\section{ACKNOWLEDGMENTS}

Work at the Los Alamos National Laboratory is performed under the auspices (Contract No. W-7405-ENG-36) of the U.S. Department of Energy. One of us (P.M.) was supported by the European TMP program LOCNET, Grant No. HPRN-CT-1999-00163.
[1] A.K. Khandpur, S. Föster, F.S. Bates, I.W. Hamley, A.J. Ryan w. Bras, K. Almdal and K. Mortensen, Macromolecules 28, 8796 (1995).

[2] M.W. Matsen, J. Phys.: Condens. Matter 14, R21 (2002).

[3] M.W. Matsen and F.S. Bates, J. Chem. Phys. 106, 2436 (1997).

[4] M.W. Matsen and F.S. Bates, Macromolecules 29, 7641 (1996).

[5] G. Riess, M. Schlienger, and S. Marti, J. Macromol. Sci., Phys. 17, 355 (1980); R. Stabler, C. Auschra, J. Beckmann, U. Krappe, I. Voigtmartin, and L. Leibler, Macromolecules 28, 3080 (1995); Y. Mogi, M. Nomura, H. Kotsuji, K. Ohnishi, Y. Matsushita, and I. Noda, ibid. 23, 6755 (1994); K. Jung, V. Abetz, and R. Stadler, ibid. 29, 1076 (1996); C.S. Patrickios, A.B. Lowe, S.P. Armes, and N.C. Billingham, J. Polym. Sci., Part A: Polym. Chem. 36, 617 (1998); Q. Ma and K.L. Wooley, ibid. 38, 4805 (2000).

[6] M. Park, C. Harrison, P.M. Chaikin, R.A. Register, and D.H. Adamson, Science 276, 1401 (1997).

[7] R.B. Thompson, V.V. Ginzburg, M.W. Matsen, and A.C. Balazs, Science 292, 5526 (2001).

[8] K. Yamauchi, H. Hasegawa, T. Hashimoto, N. Köhler, and K. Knoll, Polymer 43, 3563 (2002).
[9] K. Yamauchi, H. Hasegawa, T. Hashimoto, and M. Nagao, J. Appl. Crystallogr. 36, 708 (2003).

[10] It is important to note that our calculations and the experimental observations of Yamauchi et al., Refs. [8,9], are not directly comparable in the sense that the experiments were performed at constant temperature and the phase change was observed as function of the solvent concentration, i.e., a dynamical process, whereas our calculations pertain to equilibrium conditions only. This is the reason for us to use the "two-phase" terminology rather than the "two-step" terminology of the experimental papers.

[11] M.W. Matsen, J. Chem. Phys. 108, 785 (1998).

[12] F. Drolet and G.H. Fredrickson, Macromolecules 34, 5317 (2001).

[13] G.H. Fredrickson, V. Ganeson, and F. Drolet, Macromolecules 35, 16 (2002).

[14] H.Y. Chen and G.H. Fredrickson, J. Chem. Phys. 116, 1137 (2002).

[15] G. Tzeremes, K.Ø. Rasmussen, T. Lookman, and A. Saxena, Phys. Rev. E 65, 041806 (2002).

[16] K.Ø. Rasmussen and G. Kalosakas, J. Polym. Sci., Part B: Polym. Phys. 40, 1777 (2002). 
[17] Equal amounts of $A$ and $C$ segments were enforced by numerically requiring $\varphi_{A}(\mathbf{r})=\varphi_{C}(\mathbf{r})$ everywhere.

[18] The artificial segregation of $A$ and $C$ segments was achieved by numerically enforcing $\varphi_{A(B)}(\mathbf{r})=0$ where $\varphi_{B(A)}(\mathbf{r}) \neq 0$.

[19] The interfacial width difference is not visible in Fig. 2 because the segregation between $A$ and $C$ monomers is so weak.

[20] S. Harrington, R. Zhang, P.H. Poole, F. Sciortino, and H.E. Stanley, Phys. Rev. Lett. 78, 2409 (1997).

[21] Y. Katayama, T. Mizutani, W. Utsumi, O. Shimomura, M. Yamakata, and K. Funakoshi, Nature (London) 403, 6766 (2000). 